\title{
Comunicación Bilateral de los Nervios Músculocutáneo y Mediano. Aspectos Morfológicos e Importancia Clínica
}

\author{
Bilateral Communications of the Musculocutaneous and Median Nerves, \\ Morphological Aspects and Clinical Significance \\ *Aladino Cerda \& **Iván Suazo Galdames
}

CERDA, A. \& SUAZO, G. A. Comunicación bilateral de los nervios musculocutáneo y mediano. Aspectos morfológicos e importancia clínica. Int. J. Morphol., 30(2):651-655, 2012.

RESUMEN: Las comunicaciones entre los ramos terminales de plexo braquial son frecuentes y tienen importancia en la evaluación de traumatismos y procedimientos quirúrgicos de las regiones axilar y braquial. En este artículo presentamos un caso en el que durante la disección de rutina del miembro superior, se observó la presencia de comunicaciones bilaterales entre los nervios musculocutáneo y mediano. Los ramos comunicantes fueron descritos en su trayecto, relaciones y morfometría y se discutió acerca de la prevalencia e importancia clínica de estas comunicaciones.

PALABRAS CLAVE: Plexo braquial; nervio musculocutáneo: nervio mediano; región axilar; región braquial.

\section{INTRODUCCIÓN}

Las variaciones de los fascículos y ramos terminales del plexo braquial son relativamente comunes y han sido reportadas por varios autores (Venieratos \& Anagnostopoulou, 1998; Chauhan \& Roy, 2002; Choi et al., 2002; Arora et al., 2003; Loukas \& Aqueelah, 2005; Anyanwu et al., 2009; Maeda et al., 2009a; Maeda et al.,2009b).

El plexo braquial es una gran red nerviosa que inerva el miembro superior; comienza en el cuello y recorre el compartimento axilar (Moore \& Dalley, 2007). Está formado por la unión de los ramos anteriores de los cuatro últimos nervios cervicales (C5, C6, C7 y C8) y del primer nervio torácico (T1). El nervio musculocutáneo se origina del fascículo lateral del plexo braquial, describe un trayecto oblicuo hacia inferior y lateral, llegando a la cara medial del músculo coracobraquial, al que atraviesa en forma oblicua, encontrándose a su salida en el compartimento anterior del brazo, entre los músculos bíceps braquial y braquial (Latarjet \& Ruiz Liard, 2004). El nervio mediano se origina por dos raíces, una lateral y otra medial, que proceden cada una de los respectivos fascículos del plexo braquial. Las dos raíces convergen por anterior a la arteria axilar formando una horquilla (García-Porrero \& Hurlé, 2005).

\footnotetext{
* Universidad de Talca, Chile.

**Facultad de Medicina, Universidad Diego Portales, Santiago, Chile.
}

En el artículo relatamos un caso con múltiples comunicaciones entre los nervios musculocutáneo y mediano en el tercio superior y medio de los miembros superiores derecho e izquierdo. La incidencia de dichos ramos comunicantes en forma bilateral es poco común en la literatura (Loukas \& Aqueelah) y presenta gran variabilidad.

El propósito de este reporte fue describir la anatomía topográfica de las comunicaciones entre los nervios musculocutáneo y mediano, analizar las posibles implicancias clínicas y morfológicas.

\section{REPORTE DE CASO}

Durante una disección de rutina en un cadáver masculino de 54 años, en el laboratorio de anatomía de la Universidad de Talca, fue observada una variación anatómica bilateral en los ramos terminales del plexo braquial. Fueron disecadas las regiones axilar, pectoral y braquial, e identificados los ramos constituyentes de los nervios del plexo braquial. Los nervios musculocutáneo y mediano presentaban ramos comunicantes en ambos miembros superiores. 
En el miembro superior derecho (Fig. 1) el fascículo lateral del plexo braquial dio la raíz lateral del nervio mediano y el nervio musculocutáneo. Este último se dividió en dos ramos: uno lateral y uno medial, ambos perforaron el músculo coracobraquial. El ramo lateral continuó como nervio musculocutáneo, pasó entre los músculos bíceps braquial y braquial, inervándolos, luego siguió su trayecto distal hasta el surco bicipital lateral. El ramo medial, correspondió a un ramo comunicante que se originó a $5,8 \mathrm{~cm}$ desde el vértice del proceso coracoides, presentó un trayecto oblicuo hacia inferior y medial, con una longitud de $9,1 \mathrm{~cm}$ y un diámetro medio de $2 \mathrm{~mm}$. Finalmente incorporó se al nervio mediano a $14,9 \mathrm{~cm}$ desde el vértice del proceso coracoides.

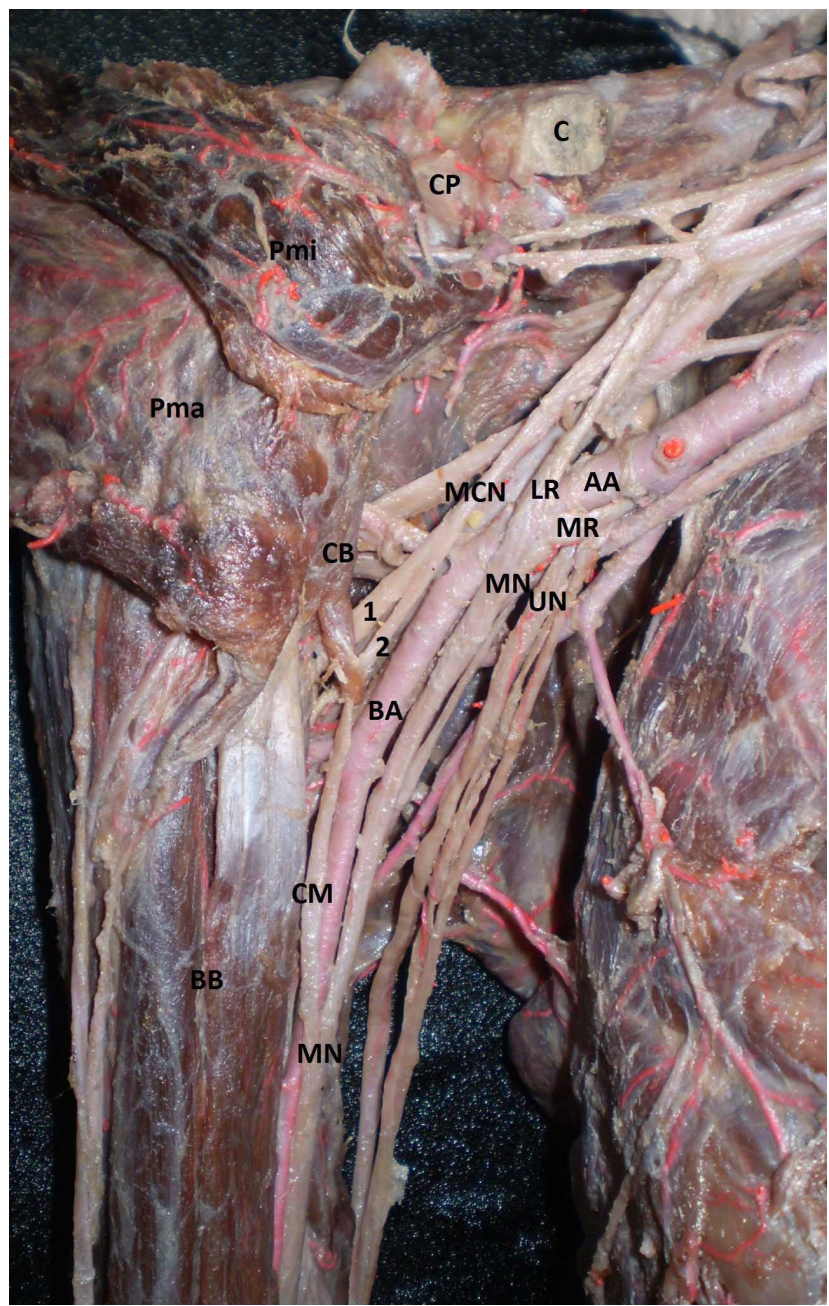

Fig. 1. Vista anterior de la región axilar derecha. C. Clavícula; CP. Proceso coracoides; Pma. Músculo pectoral mayor; Pmi. Músculo pectoral menor; CB. Músculo coracobraquial; MCN. Nervio musculocutáneo; AA. Arteria axilar; LR. Raíz lateral del nervio mediano; MR. Raíz medial del nervio mediano; 1. Ramo lateral del nervio musculocutáneo; 2. Ramo medial del nervio musculocutáneo; BA. Arteria braquial; CMT. Ramo comunicante; BB. Músculo bíceps braquial; MN. Nervio mediano.
El fascículo medial del plexo braquial originó la raíz medial del nervio mediano, que se unió a la raíz lateral por anterior a la arteria axilar.

En el miembro superior izquierdo (Fig. 2) el fascículo lateral del plexo braquial originó la raíz lateral del nervio mediano y continuó como un tronco, el cual se dividió en un ramo comunicante proximal a $5,5 \mathrm{~cm}$ desde el vértice del proceso coracoides, y que cruzó superficialmente a la arteria axilar, con una longitud y un diámetro similar a la raíz lateral del nervio mediano (longitud de $3,7 \mathrm{~cm}$ y un diámetro de $2 \mathrm{~mm}$ ), y se unió al nervio mediano a 5,9 cm desde el vértice del proceso coracoides. El resto del fascículo lateral continuó como nervio musculocutáneo. El nervio musculocutáneo perforó el músculo coracobraquial, pasó entre los músculos bíceps braquial y braquial, dando ramos

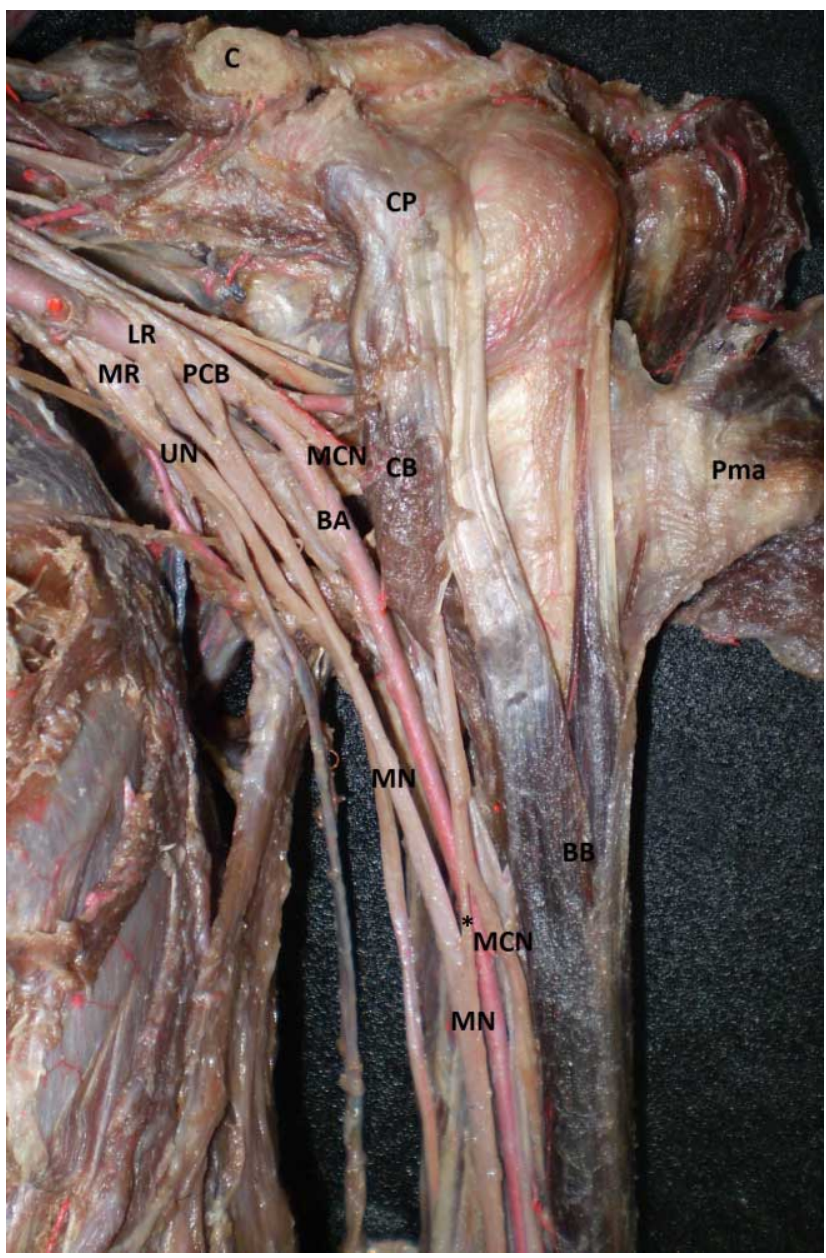

Fig. 2. Vista anterior de la región axilar izquierda. Clavícula; CP. Proceso coracoides; Pma. Músculo pectoral mayor; AA. Arteria axilar; LR. Raíz lateral del nervio mediano; MR. Raíz medial del nervio mediano; PCB. Ramo comunicante proximal; UN. nervio ulnar; MCN. Nervio musculocutáneo; CB. Músculo coracobraquial; BA. Arteria braquial; BB. Músculo bíceps braquial; MN. Nervio mediano; *: Ramo comunicante distal. 
a los tres músculos y emitió un ramo comunicante distal, que nació a 13,6 cm desde el vértice del proceso coracoides, siguió una dirección oblicua hacia inferior y medial, con una longitud de $1,9 \mathrm{~cm}$ y un diámetro de $1 \mathrm{~mm}$ y se unió al nervio mediano a $15,3 \mathrm{~cm}$ del vértice del proceso coracoides. Luego continuó su descenso hasta el surco bicipital lateral. $\mathrm{Al}$ igual que en el miembro superior derecho, las raíces lateral y medial del nervio mediano confluyeron por anterior a la arteria axilar.

\section{DISCUSION}

Las variaciones anatómicas del plexo braquial pueden ser explicadas a través de su desarrollo embriológico. El esbozo del miembro superior se puede observar hacia el día 26-27 en el embrión en desarrollo. Las fibras nerviosas motoras que surgen de la médula espinal comienzan a aparecer a finales de la cuarta semana (Moore \& Persaud, 2008), mientras que las uniones neuromusculares se desarrollan entre las semanas 12 y 20 (Zagrebin \& Chuchkov, 1980). El plexo braquial aparece como un cono radicular único en el miembro superior. Este cono se divide longitudinalmente en segmentos ventral y dorsal y durante la migración axonal, pueden ocurrir comunicaciones atípicas.

La comprensión de la complejidad de la formación y estructura del plexo braquial sigue siendo una piedra angular para el manejo quirúrgico efectivo y de anestesia general. El conocimiento de las posibles variaciones en su formación y estructura es importante tanto para anatomistas como para médicos (Johnson et al., 2010). Las variaciones en los ramos terminales del plexo braquial son frecuentes y han sido reportadas por diversos autores (Venieratos \& Anagnostopoulou; Olave et al., 2000; Chauhan \& Roy; Choi et al., ; Arora et al.,; Loukas \& Aqueelah; Anyanwu et al.). La variación más frecuente consiste en la presencia de un ramo comunicante que se bifurca desde el nervio musculocutáneo y va distalmente para unirse al nervio mediano, a nivel del tercio inferior del brazo (Venieratos \& Anangnostopoulou). Si este ramo se desprende en el tercio superior del brazo es generalmente considerado como la segunda raíz lateral del nervio mediano (Chauhan \& Roy). De acuerdo con Saeed \& Rufai (2003), estas variaciones son predominantemente unilaterales. Choi et al. señalan que las variaciones bilaterales son muy raras.

Las comunicaciones entre los nervios musculocutáneo y mediano han sido clasificadas por muchos autores en base a diferentes criterios. Choi et al. clasificaron las comunicaciones entre el nervio musculocutáneo y el nervio mediano en tres patrones en base al número de ramos comunicantes o la fusión de ambos nervios. En el patrón I los nervios musculocutáneo y mediano se fusionan formando un tronco. En el patrón 2 existe un ramo comunicante entre el nervio musculocutáneo y mediano y en el patrón 3 dos ramos comunicantes estaban presentes entre los nervios musculocutáneo y mediano. Según esta clasificación, nuestras observaciones pueden ser clasificadas como un patrón II en el miembro superior derecho y III en el izquierdo. Loukas \& Aqueelah clasificaron las comunicaciones entre el nervio mediano y musculocutáneo en 4 tipos en relación con el músculo coracobraquial. En los tipos I y II las comunicaciones son proximal y distal al punto de ingreso del nervio musculocutáneo en el coracobraquial, respectivamente. En el tipo III el nervio musculocutáneo no perfora el coracobraquial y en el tipo IV coexisten los tipos I y II. De acuerdo con esta clasificación el miembro superior derecho corresponde al tipo II, mientras que el miembro superior izquierdo corresponde al tipo IV. La coexistencia de los tipos II y IV sólo fue encontrada en un individuo $(0,8 \%$ de los casos) (Loukas \& Aqueelah). En la mayoría de los casos reportados por Choi et al., los ramos comunicantes fueron desde el nervio musculocutáneo al mediano al igual que todos las comunicaciones observadas en nuestro caso.

En cuando al ramo comunicante proximal en el miembro superior izquierdo. Este ya había sido descrito en estudios previos como una segunda raíz lateral del nervio mediano (o tercera raíz) (Uzun \& Seelig, 2001; Saeed \& Rufai; Goyal et al., 2005; Nene et al., 2010), quienes lo asociaron con un ramo comunicante anómalo entre los nervios musculocutáneo y mediano. Dicho ramo comunicante también fue observado en nuestro caso (ramo comunicante distal), pero al contrario de Saeed \& Rufai, el ramo comunicante distal se originaba desde el nervio musculocutáneo al mediano. En otros estudios esta segunda raíz lateral fue clasificada como una comunicación entre los nervios musculocutáneo y mediano proximal al músculo coracobraquial (Venieratos \& Anagnostopoulou; Loukas \& Aqueelah). Según Singhal et al. (2007), los ramos anómalos del fascículo lateral, como lo es la segunda raíz lateral del nervio mediano, que cruzan la arteria axilar anteriormente pueden causar síndromes de compresión produciendo isquemia. Este tipo de variación es importante, ya que es más propenso a lesionarse en las operaciones quirúrgicas de la axila y miembro superior (Chauhan \& Roy).

El plexo braquial suministra la inervación cutánea y muscular al miembro superior y cualquier lesión a este nivel puede llevar a una discapacidad significativa (Villamere et al., 2009). Tanto la posición normal y anormal de las arterias y venas se puede determinar antes de la operación por estudios angiográficos, pero en caso de los nervios no es posible detectar este tipo de anomalía. Sólo en el momento 
de la cirugía el cirujano está expuesto a tales variaciones (Das \& Paul, 2005). La presencia de variaciones anatómicas es utilizada frecuentemente para explicar los síntomas que podrían no ser obvios de otra manera (Loukas et al., 2008). Uzún \& Seelig señalan que este tipo de variaciones son vulnerables a los daños en la disección radical de cuello y en otras operaciones quirúrgicas en la fosa axilar y parte superior del brazo. En nuestro caso dos ramos comunicantes entre los nervios musculocutáneo y mediano, se encontraban en el tercio medio del brazo, uno en el derecho y otro en el izquierdo. Este tipo de comunicación es importante durante el abordaje anterior de la fractura del húmero (Chitra, 2007). Dichos ramos comunicantes, fueron distales al músculo coracobraquial. En el miembro superior derecho, el ramo comunicante perforaba el músculo coracobraquial a pesar de ser emitido desde el nervio musculocutáneo antes de perforar el músculo. Este tipo de comunicación puede ser relacionada con síndromes de compresión. La compre- sión del nervio musculocutáneo es rara y se puede producir después de un ejercicio físico vigoroso (Yimaz et al., 2005) o en los movimientos violentos pasivos del brazo o antebrazo (Kim \& Goodrich, 1984). Si esta situación coexiste con una unión tipo II, entonces gran parte del nervio mediano pasa también por el músculo coracobraquial. En este caso el síndrome de compresión podría incluir síntomas similares a los encontrados en la neuropatía del nervio mediano. Este conocimiento podría ser útil para el clínico, con el fin de evitar una liberación innecesaria del túnel carpiano (Venieratos \& Anagnostopoulou).

Considerando la alta frecuencia de las intervenciones quirúrgicas del miembro superior es esencial estar al tanto de las variaciones anatómicas debido a su vulnerabilidad. Este tipo de comunicaciones nos hacen recordar la particularidad de cada paciente y la importancia de la individualización de los tratamientos médicos o quirúrgico.

CERDA, A. \& SUAZO, G. A. Bilateral communications of the musculocutaneous and median nerves. Morphological aspects and clinical significance. Int. J. Morphol., 30(2):651-655, 2012.

SUMMARY: Communication of the musculocutaneous and median nerves of the brachial plexus is common and is important in the evaluation of trauma and surgical procedures in axillary and brachial regions. This paper presents a case in which during a routine dissection of upper limb the presence of bilateral communication between musculocutaneous and medium nerves was observed. Trajectory of communicating branches was described, relation and morphometry was discussed with regard to prevalence and clinical significance of these communications.

KEY WORDS: Brachial plexus; musculocutaneous nerve, median nerve; axillary region; brachial region.

\section{REFERENCIAS BIBLIOGRAFICAS}

Anyanwu, G.; Obikili, E.; Esom, A. \& Ozoemana, F. Prevalence and pattern of communication of medial and musculocutaneous nerves within the black population: Nigeria - a case study. Int. J. Biomedical and Health Sciences, 5(2):87-94, 2009.

Arora, J.; Kapur, V.; Suri, R.K. \& Khan, R.Q. Inter-Communications Between Median And Musculocutaneous Nerves With Dual Innervation of Brachialis Muscle-A Case Report. J. Anat. Soc. India, 52(1):66-8, 2003.

Chauhan, R. \& Roy, T.S. Communication Between The Median And Musculocutaneous Nerve-A Case Report. J Anat. Soc. India, 51(1):72-5, 2002.

Chitra, R. Multiple bilateral neuroanatomical variations of the nerves of the arm. Neuroanatomy, 6:43-45, 2007.

Choi, D.; Rodríguez, M.; Vázquez, T.; Parkin, I. \& Sañudo, J.R. Patterns of Connections Between the Musculocutaneous and Median Nerves in the Axilla and Arm. Clin. Anat., 15:11-17, 2002.
Das, S.; Paul, S. Anomalous Branching Pattern of Lateral Cord of Brachial Plexus. Int J. Morphol., 23(4):289-292, 2005.

García-Porrero, J. \& Hurlé, J. Anatomía humana. 1a. ed. Madrid: Mc Graw-Hill Interamericana, 2005.

Goyal, N.; Harjeet.; Gupta, M. Bilateral variant contributions in the formation of median nerve. Surg. Radiol. Anat., 27:562$565,2005$.

Johnson, E.O; Vekris, M.; Demesticha, T.; Soucacos, P.N. Neuroanatomy of the brachial plexus: normal and variant anatomy of its formation. Surg .Radiol. Anat., 32:291-7, 2010.

Kim, S.M \& Goodrich, J.A. Isolated proximal musculocutaneous nerve palsy: case report. Arch. Phys. Med. Rehabil., 65(11):735$6,1984$.

Latarjet, M. \& Ruíz Liard, A. Anatomía humana. 4a. ed. Buenos Aires: Médica Panamericana, 2004. 
CERDA, A. \& SUAZO, G. A. Comunicación bilateral de los nervios musculocutáneo y mediano. Aspectos morfológicos e importancia clínica. Int. J. Morphol., 30(2):651-655, 2012.

Loukas, M. \& Aqueelah, H. Musculocutaneous and median nerve connections within, proximal and distal to the coracobrachialis muscle. Folia Morphol,, 64(2):101-10, 2005.

Loukas, M.; Tubbs, R.S.; Stewart, D. An Abnormal Variation of the Brachial Plexus with Potential Clinical Significance. West Indian Med. J., 57(4):403-405, 2008.

Maeda, S.; Kawai, K.; Koizumi, M.; Ide, J.; Tokiyoshi, A.; Mizuta, H., et al. Morphological study of the communication between the musculocutaneous and median nerves. Anat. Sci. Int., 84:34-40, 2009a.

Maeda, S.; Kawai, K.; Koizumi, M.; Ide, J.; Tokiyoshi, A.; Mizuta, $\mathrm{H}$., et al. Morphological study, by teasing examination, of the communication from the musculocutaneous to median nerves. Anat. Sci. Int., 84:41-46, 2009b.

Moore, K. \& Dalley, A. Anatomía con orientación clínica. 5a. ed. México: Editorial Médica Panamericana, 2007.

Moore, K. \& Persaud, T. Embriología clínica. 8a. ed. Madrid: Elsevier, 2008.

Nene, A.; Gajendra, K.; Sarma, M. A rare variant formation of the median nerve. Int. J. . Anatomical Variations, 3:138-40, 2010.

Olave, E.; Gabrielli, C.; Braga, M.T.T.; Del Sol, M.; De Souza, A. Ramo comunicante entre los nervios musculocutáneo y mediano en el hombre. Rev. Chil. Anat, 18(2):301-4, 2000.

Saeed, M. \& Rufai, A. Median and Musculocutaneous Nerves: Variant Formation and Distribution. Clin. Anat., 16:453-457, 2003.

Singhal, S.; Vijay, V.; Ravindranath, R. Variations in brachial plexus and the relationship of median nerve with the axillary artery: a case report. J. Brachial Plexus and Peripheral Nerve Injury., $2: 21,2007$.

Uzún, A. \& Seelig, L.L. A variation in the formation of the median nerve: communicating branch between the musculocutaneous and median nerves in man. Folia Morphol., 60(2):99-101, 2001.

Venieratos, D. \& Anagnostopoulou, S. Classification of Communications Between the Musculocutaneous and Median Nerves. Clin. Anat., 11:327-31, 1998.

Villamere, J.; Goodwin, S.; Hincke, M.; Jalali A. A brachial plexus variation characterized by the absence of the superior trunk. Neuroanatomy, 8: 4-6, 2009.

Yilmaz, C.; Eskandari, M.M; Colak, M. Traumatic musculocutaneous neuropathy: a case report. Arch. orthopaedic and trauma surg., 125(6):414-6, 2005.

Zagrebin AM, Chuchkov VM. Formation of the neurovascular connections in human forearm muscles. Arkh. Ana.t Gistol. Embriol., 79:67-73, 1980.

\section{Correspondence To \\ Prof. Dr. Iván Suazo Galdames \\ Facultad de Medicina \\ Universidad Diego Portales \\ Santiago \\ CHILE}

Email: ivan.suazo@udp.cl

Received: 07-09-2011

Acceped : 18-02-2012 